
\title{
.
}

\section{Polyamine Depletion Attenuates Isoproterenol-Induced Hypertrophy and Endoplasmic Reticulum Stress in Cardiomyocytes}

\author{
Yan Lina,b,d Xiaojie Zhanga Lina Wang ${ }^{c}$ Yajun Zhaoc Hongzhu Lic Wei Xiaob \\ Changqing $\mathrm{Xu}^{\mathrm{c}}$ Jicheng $\mathrm{Liu}^{\mathrm{a}}$ \\ anstitute of medicine, Qiqihar Medical University, Qiqihar, ${ }^{\mathrm{b} D e p a r t m e n t}$ of Pathophysiology, Qiqihar \\ Medical University, Qiqihar, 'Department of Pathophysiology, Harbin Medical University, Harbin, \\ dTCWM Postdoctoral Research Center in Heilongjiang University of Traditional Chinese Medicine, \\ Harbin, PR China
}

\section{Key Words}

Cardiac hypertrophy $\bullet$ Apoptosis $\bullet$ Polyamine $\bullet$ Endoplasmic reticulum stress

\begin{abstract}
Background/Aim: Polyamines (putrescine, spermidine and spermine) play an essential role in cell growth, differentiation and apoptosis. Hypertrophy is accompanied by an increase in polyamine synthesis and endoplasmic reticulum stress (ERS) in cardiomyocytes. The present study was undertaken to elucidate the molecular interactions between polyamines, ERS and cardiac hypertrophy. Methods: Myocardial hypertrophy was simulated by incubating cultured neonatal rat cardiomyocytes in $100 \mathrm{nM}$ isoproterenol (ISO). Polyamine deletion was achieved using $0.5 \mathrm{mM}$ difluoromethylornithine (DFMO). Hypertrophy was estimated using cell surface area measurements, total protein concentrations and atrial natriuretic peptide (ANP) gene expression. Apoptosis was measured using flow cytometry and transmission electron microscopy. Expression of ornithine decarboxylase (ODC) and spermidine/spermine N1-acetyltransferase (SSAT) were analyzed via real-time PCR and Western blotting. Protein expression of ERS and apoptosis factors were analyzed using Western blotting. Results: DFMO $(0.5 \mathrm{mM}$ and $2 \mathrm{mM})$ treatments significantly attenuated hypertrophy and apoptosis induced by ISO in cardiomyocytes. DFMO also decreased lactate dehydrogenase (LDH) and malondialdehyde (MDA) level in the culture medium. In addition, DFMO $(0.5 \mathrm{mM})$ down regulated the expression of ODC, glucose-regulated protein 78 (GRP78), C/EBP homologous protein (CHOP), cleaved caspase-12, and Bax and up regulated the expression of SSAT and $\mathrm{Bcl}-2$. Finally, these changes were partly reversed by the addition of exogenous putrescine $(0.5$ $\mathrm{mM}$ ). Conclusion: The data presented here suggest that polyamine depletion could inhibit cardiac hypertrophy and apoptosis, which is closely related to the ERS pathway.

Y. Lin and X. Zhang contributed equally.




\section{Introduction}

Polyamines (spermine, spermidine and putrescine) are polycationic aliphatic amines that influence nucleic acid structure and stability, modulate ion channel activity, govern mRNA translation rates, and are important mediators of cellular growth and division. In cardiomyocytes, increasing evidence indicates that polyamines play a role in various aspects of cardiac disease $[1,2]$. Ornithine decarboxylase (ODC) is the enzyme that decarboxylates ornithine to the diamine putrescine, the precursor of spermidine and spermine and the ratelimiting step in polyamine biosynthesis in mammalian cells [3]. Inhibition of ODC, using the ornithine analog difluoromethylornithine (DFMO), has proved an effective means of depleting cellular polyamines in mammals and is clinically approved for humans.

The endoplasmic reticulum (ER) is an organelle that is responsible for regulating protein synthesis, protein folding, trafficking and intracellular calcium levels $[4,5]$. Accumulation of misfolded or unfolded proteins in the ER can result in endoplasmic reticulum stress (ERS) [6]. In the early stages of ERS, a series of signaling pathways assist in protein folding, severe or prolonged ERS will trigger cellular apoptosis. C/EBP homologous protein (CHOP) is an important transcription factor that mediates protein kinase R-like ER kninase (PERK) activation-induced apoptosis in ERS [7]. Caspase-12 mediated apoptosis is a specific apoptotic pathway in the ER, which is independent on mitochondria or death receptor activation. ERS is required for proper cardiac development, and it provides certain protective mechanisms against cardiac damage caused by various stresses, as well as being involved in cardiovascular pathological processes, such as myocardial infarction, ischemia/ reperfusion and pressure overload-induced hypertrophy [8-10].

Cardiac hypertrophy, an enlargement of the heart, is a major risk factor that is associated with adverse cardiovascular outcomes [11]. Although our previous studies demonstrated that DFMO can attenuate cardiomyocyte hypertrophy by inhibiting the NO/cGMP-dependent protein kinase-1 pathway, no study, to date, has directly addressed the effect of DFMO on ERS and the underlying mechanisms in cardiac hypertrophy $[12,13]$. We hypothesize that the protective effects of DFMO on cardiomyocytes may be at least partially due to ERS.

\section{Materials and Methods}

\section{Materials}

Isoproterenol (ISO), DFMO, putrescine (Pu) and trypsinase were purchased from Sigma-Aldrich (St. Louis, MO, USA). ODC, spermidine/spermine N1-acetyltransferase (SSAT), glucose-regulated protein 78 (GRP78), CHOP, caspase-12, Bax and Bcl-2 primary antibodies were obtained from Santa Cruz Biotechnology Incorporated (Santa Cruz, CA, USA). Trizol reagent and the PrimerScript RT reagent kit were purchased from TakaRa Biotechnology Incorporated (TakaRa Bio Inc, Japan). The Western blot kit and $\beta$-actin antibody were purchased from Boster (Wuhan, China). High glucose Dulbecco's modified Eagle's medium (DMEM) was purchased from Gibco BRL (Gaithersburg, MD, USA). Assay kits for malondialdehyde (MDA) and lactate dehydrogenase (LDH) were purchased from Jiancheng Bioengineering Institute (Nanjing, China)

\section{Cardiomyocytes culture and treatment}

Primary cultures of neonatal rat cardiomyocytes were established and maintained as previously described [14]. Neonatal rat cardiomyocytes were prepared from 2- to 3-day-old neonatal Wister rats. The rats were handled in accordance with the Guide for the Care and Use of Laboratory Animals, published by the China National Institutes of Health. Briefly, the heart were minced and dissociated with $0.25 \%$ trypsinase. Dispersed cells were seeded at $2 \times 10^{5} \mathrm{cells} / \mathrm{cm}^{2}$, in $60-\mathrm{mm}$ culture dishes, with DMEM supplemented with $10 \%$ fetal bovine serum (FBS), and then cultured in a $5 \% \mathrm{CO}_{2}$ incubator at $37^{\circ} \mathrm{C}$.

\section{Treatment protocol}

Three days after the cells were seeded, the cardiomyocytes were randomly divided into the following four groups: (1) control group: cardiomyocytes were continuously cultured for $72 \mathrm{~h}$ in DMEM; (2) ISO 
group: cardiomyocytes were treated with $100 \mathrm{nM}$ ISO for $48 \mathrm{~h}$; (3) pre-DFMO group: cardiomyocytes were pre-incubated with $0.1 \mathrm{mM}, 0.5 \mathrm{mM}$ or $2 \mathrm{mM}$ DFMO for $24 \mathrm{~h}$, and then treated with $100 \mathrm{nM}$ ISO for $48 \mathrm{~h}$; (4) pre-(DFMO+Pu) group: cardiomyocytes were pre-incubated with $0.5 \mathrm{mM}$ DFMO and $0.5 \mathrm{mM}$ Pu for 24 $\mathrm{h}$, and then treated with $100 \mathrm{nM}$ ISO for $48 \mathrm{~h}$. All drugs were dissolved in the pre-warmed culture medium and added directly to the cardiomyocytes. In control groups, equivalent volumes of medium were added.

Measurement of the surface area and total protein concentrations of cardiomyocytes

Cardiomyocytes were counted from a minimum of three dishes per group using phase-contrast microscopy. The cellular surface area was measured by the Image Analysis System (Olympus). Ten fields were randomly chosen for each group, and 10 cardiomyocytes randomly chosen for each field. The total protein concentrations was measured by Bradford's method in each group.

\section{Assessment of apoptosis}

Apoptosis was evaluated using double fluorescence staining with annexin V/propidiumiodide (PI). Cardiomyocytes $\left(1 \times 10^{5}\right.$ cells per well) were pre-treated with DFMO and DFMO+Pu for $24 \mathrm{~h}$, and then incubated with ISO (100 nM) for $48 \mathrm{~h}$. After treatment, cardiomyocytes were washed three times with icecold PBS, and then treated with $5 \mathrm{~mL}$ PI and $10 \mathrm{~mL}$ annexin V-FITC (Jingmei Biotech, Shanghai, China) at room temperature for $10 \mathrm{~min}$ in the dark. Apoptosis of cardiomyocytes was examined by flow cytometer (Becton, Dickinson and Company, USA) within $1 \mathrm{~h}$.

\section{Transmission electron microscopy}

Cardiomyocytes were harvested and fixed with 3.0\% glutaraldehyde and 1.5\% paraldehyde, washed in phosphate buffer saline (PBS), and fixed in osmium tetroxide. Cells were then dehydrated in an ethanol series, embedded in epoxy resin and examined under a transmission electron microscope (Hitachi, TEMHT7700).

\section{Real time-PCR analysis of ANP, ODC and SSAT}

Total RNA was extracted from the cardiomyocytes using the phenol guanidine isothiocyanate method (Trizol kit, TaKaRa) as per the manufacturer's instructions. The RNA was reverse transcribed with oligodT and Superscript First-Strand Synthesis System for RT-PCR (TaKaRa), according to the manufacturer's instructions. The cDNA was then amplified using an iCycler (ABI) and the Brilliant SYBR Green QPCR master mix (TaKaRa). Relative quantification of gene expression was normalized using $\beta$-actin as the control gene. The nucleotide sequences of the primers used are: (1) ANP: sense 5-GGGAAGTCAACCCGTCTCA-3, antisense5-GGGCTCCAATCCTGTCAAT-3; (2) ODC: sense 5-GCTTTCTATGTTGCGGACCT-3, antisense 5-TGCTCACTATGGCTCTGCTG-3, (3) SSAT: sense 5-GTGAGCATCCATTCCAAAGC-3, antisense 5-ATTCTGCCTCCAAACCACAT-3.

Western blotting analysis of ODC, SSAT, GRP78, CHOP, caspase-12, Bcl-2, and Bax

Total proteins were prepared from neonatal rat cardiomyocytes as described previously [15]. Total protein samples $(30 \mu \mathrm{g}$ ) were loaded in to $10 \%$ sodium dodecyl sulfate-polyacrylamide gels, electrophoresed, and then transferred to a polyvinylidene fluoride membrane. Membranes were blocked with 5\% skimmed milk in Tris-buffered saline, with $0.1 \%$ Tween 20 (TBST) for $1 \mathrm{~h}$ at room temperature, and then incubated overnight at $4^{\circ} \mathrm{C}$ with primary antibody ODC (1:500), SSAT (1:500), GRP78 (1:1000), CHOP (1:500), caspase-12 (1:1000), Bcl-2 (1:500) and Bax (1:500). The membrane was next washed three times in 1× TBST, and incubated with anti-IgG antibody conjugated with peroxidase (1:5000) in TBST for $1 \mathrm{~h}$ at room temperature. Using an enhanced chemiluminescence detection kit (Pierce Chemical Company, Rockford, IL, USA). $\beta$-actin expression was used as the control.

\section{Measurement of LDH activity and MDA content}

The level of MDA in the culture medium was measured using a commercial thiobarbituric acid-reactive substances assay kit (Jiancheng Bioengineering Institute, Nanjing, China), according to the manufacturer's instructions. MDA values were expressed as nmole per gram of protein. The activity of lactate dehydrogenase (LDH) in the culture medium, as an indicator of cytotoxicity, was measured spectrophotometrically using a commercially available assay kit (Jiancheng Bioengineering Institute, Nanjing, China). 
Fig. 1. Hypertrophic state in neonatal rat cardiomyocytes. Cardiomyocytes were pretreated with DFMO (0.1 mM, 0 . $5 \mathrm{mM}$ and $2 \mathrm{mM}$ ) or $0.5 \mathrm{mM}$ $(\mathrm{DFMO}+\mathrm{Pu})$ for $24 \mathrm{~h}$, and then treated with $100 \mathrm{nM}$ ISO. Data are means \pm S.E.M. of four determinations. ${ }^{*} P$ $<0.05$ or ${ }^{* *} P<0.01$ versus control group; ${ }^{\#} P<0.05$ or ${ }^{\# \#} P<0.01$ versus ISO group; $\wedge$ $P<0.05$ or $\triangle \wedge P<0.01$ versus pre-DFMO (0.5 mM) group. (A) The surface area of cardiomyocytes. (B) The total protein concentrations of cardiomyocytes. (C) The gene expression of ANP determined by real-time PCR.

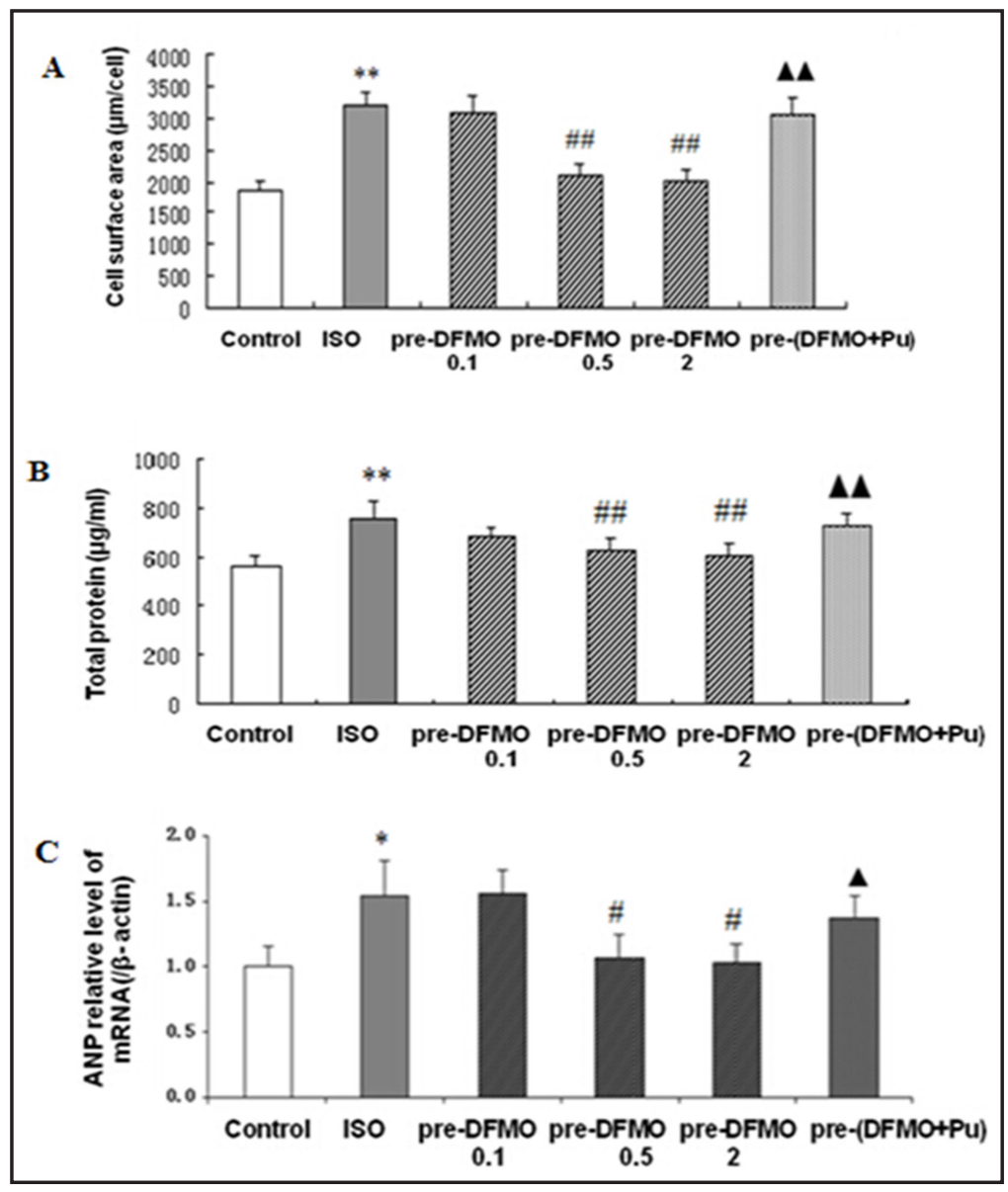

Statistical analysis

Data from at least three independent experiments for each condition were collected. Values are means \pm S.E.M. Comparisons among the groups were carried out using Kruskal-Wallis one-way ANOVA with $P<$ 0.05 considered to be statistically significant.

\section{Results}

Hypertrophic occurrences in neonatal rat cardiomyocytes

To investigate the effect of DFMO on hypertrophy, cardiomyocytes were treated with ISO, DFMO and putrescine. Cardiomyocyte hypertrophy was evaluated by the following; namely surface area of cardiomyocytes, total protein concentrations and mRNA expression of ANP (a cardiac hypertrophy marker gene). The results showed ISO increased surface area $(P<0.01)$, total protein concentrations $(P<0.01)$, and up regulated mRNA expression of ANP $(P<0.05)$ compared with the control group. In contract, after DFMO treatment $(0.5 \mathrm{mM}$ and $2.0 \mathrm{mM}$ ), hypertrophy markers were significantly decreased $(P<0.01$ or $P<0.05$ versus ISO group). Pretreatment with $0.1 \mathrm{mM}$ DFMO had no effect on hypertrophy. In the pretreated 0.5 $\mathrm{mM}$ DFMO and $0.5 \mathrm{mM}$ Pu group, hypertrophy increased relative to the DFMO treatment $(P$ $<0.01$ or $P<0.05$ versus DFMO group)( Fig. $1 \mathrm{~A}-\mathrm{C}$ ).

DFMO inhibited ISO-induced apoptosis of cardiomyocytes

In apoptotic cells, the membrane phosphatidylserine translocates from the inner to the outer surface of the plasma membrane, while the membrane remains physically intact. Apoptotic cells were stained with annexin V-fluorescein isothiocyanate (FITC), which binds 
Fig. 2. Effect of DFMO on cardiomyocytes apoptosis induced by ISO. Cardiomyocytes were pretreated with $0.5 \mathrm{mM}$ DFMO or $0.5 \mathrm{mM}$ (DFMO + Pu) for 24 $\mathrm{h}$, and then treated with $100 \mathrm{nM}$ ISO. Apoptosis was measured by annexin V/PI staining (flow cytometric analysis). ${ }^{*} P<0.05$ versus control group; ${ }^{\#} P<0.05$ versus ISO group; $\triangle P<0.05$ versus preDFMO group.
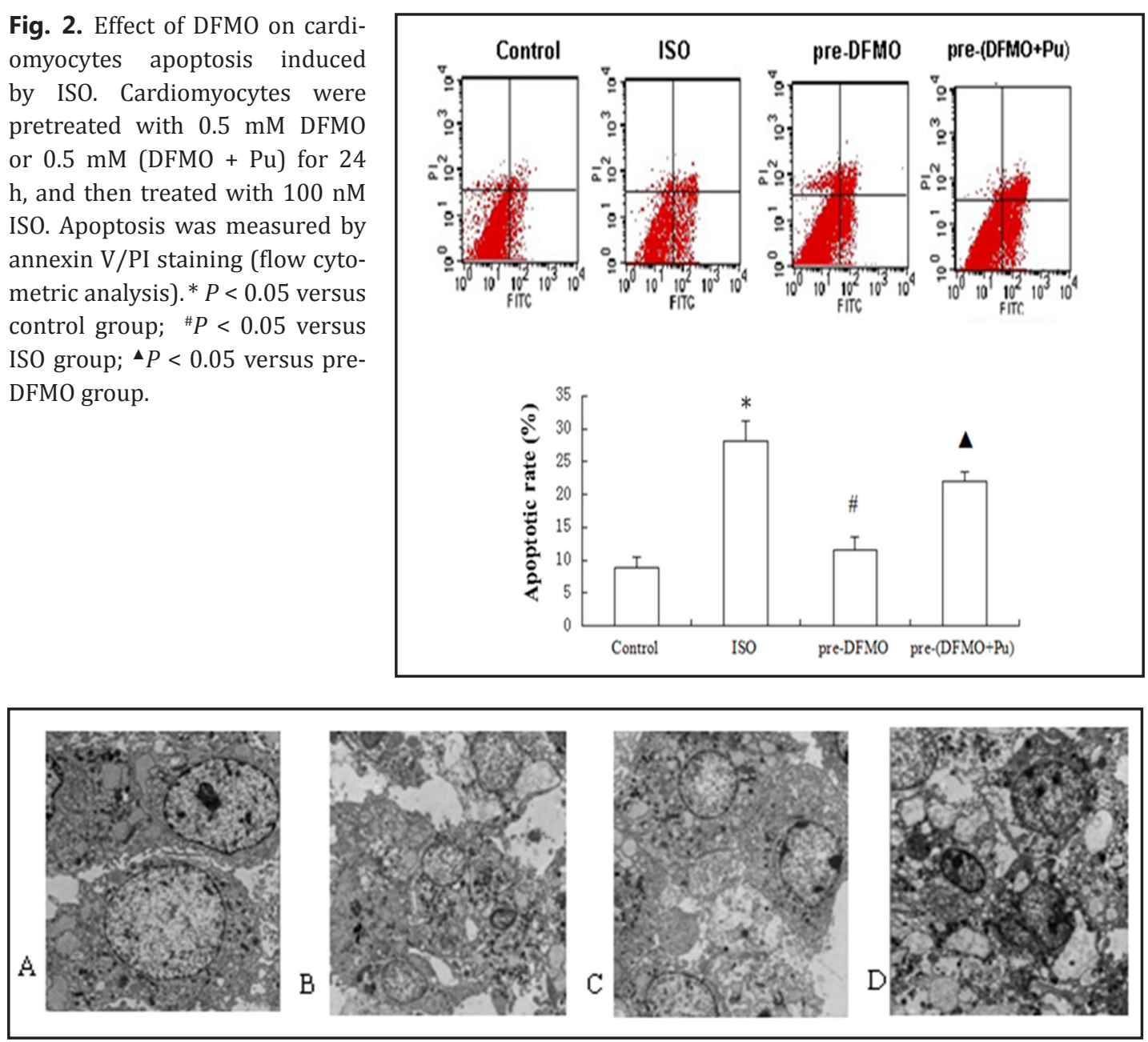

Fig. 3. Ultrastructural changes in cardiomyocytes. (A) Control group (B) ISO group (C) $0.5 \mathrm{mM}$ pre-DFMO group (D) $0.5 \mathrm{mM}$ pre-(DFMO $+\mathrm{Pu})$ group (magnification $\times 8000)$. In the ISO and pre-(DFMO $+\mathrm{Pu})$ group, nuclear chromatin margination, aggregation, and condensation and mitochondrial swelling and vacuolisation were observed. There was no significant change in nuclear chromatin in the control group. Cellular injury was lessened in the pre-DFMO group when compared with the ISO group.

phosphatidylserine with high affinity, resulting in green fluorescence when excited at 620 $\mathrm{nm}$. Necrotic cells loose the physical integrity of their plasma membrane and are able to be stained with both annexin V-FITC and PI. Living cells remain unstained. Flow cytometry analysis detected minimal apoptosis in the control group, the basal early apoptotic rate was $8.92 \%$. Apoptosis significantly increased to $28.05 \%$ when cells were exposed to ISO $(P<$ 0.05 versus control group). The $0.5 \mathrm{mM}$ DFMO treatment group demonstrated decreased apoptosis $(11.64 \%)$ compared to the ISO group ( $P<0.05$ versus ISO group). In contrast, the $0.5 \mathrm{mM}$ DFMO and $0.5 \mathrm{mM}$ Pu treatment group showed an increase in apoptosis relative to the DFMO treatment group (21.93\%) ( $P<0.05$ versus pre-DFMO group) (Fig. $2 \mathrm{~A}$ and $2 \mathrm{~B}$ ).

\section{The effect of DFMO on morphological changes of cardiomyocytes}

The results of transmission electron microscopy showed that the nuclear membrane was intact, even distribution of nuclear chromatin, and intact mitochondria structures in the control group. Morphological changes characteristic of apoptosis, including nuclear chromatin margination, aggregation, and condensation, as well as swelling and vacuolisation of mitochondria were observed in the ISO and pre-(DFMO+Pu) treatment groups. Compared 
Fig. 4. Effect of DFMO on ODC and SSAT gene and protein levels in cardiomyocytes. ODC and (B) SSAT protein levels as shown by Western blot. The intensity of each band was quantified by densitometry, and data were normalized to $ß$ actin. (C) ODC and SSAT gene expression as determined by real-time PCR. Control group expression levels were considered the basal levels, and data from other experimental groups are expressed as fold change from the control group. The fold change values represent the mean \pm S.E.M. of four determinations. ${ }^{*} P<0.05$ or ${ }^{* *} P<0.01$ versus control group. ${ }^{\#} P$ $<0.05$ or ${ }^{\# \# ~} P<0.01$ versus ISO group. ${ }^{\star} P<0.05$ or $\triangle \Delta P<0.01$ versus 0.5 $\mathrm{mM}$ pre-DFMO group.

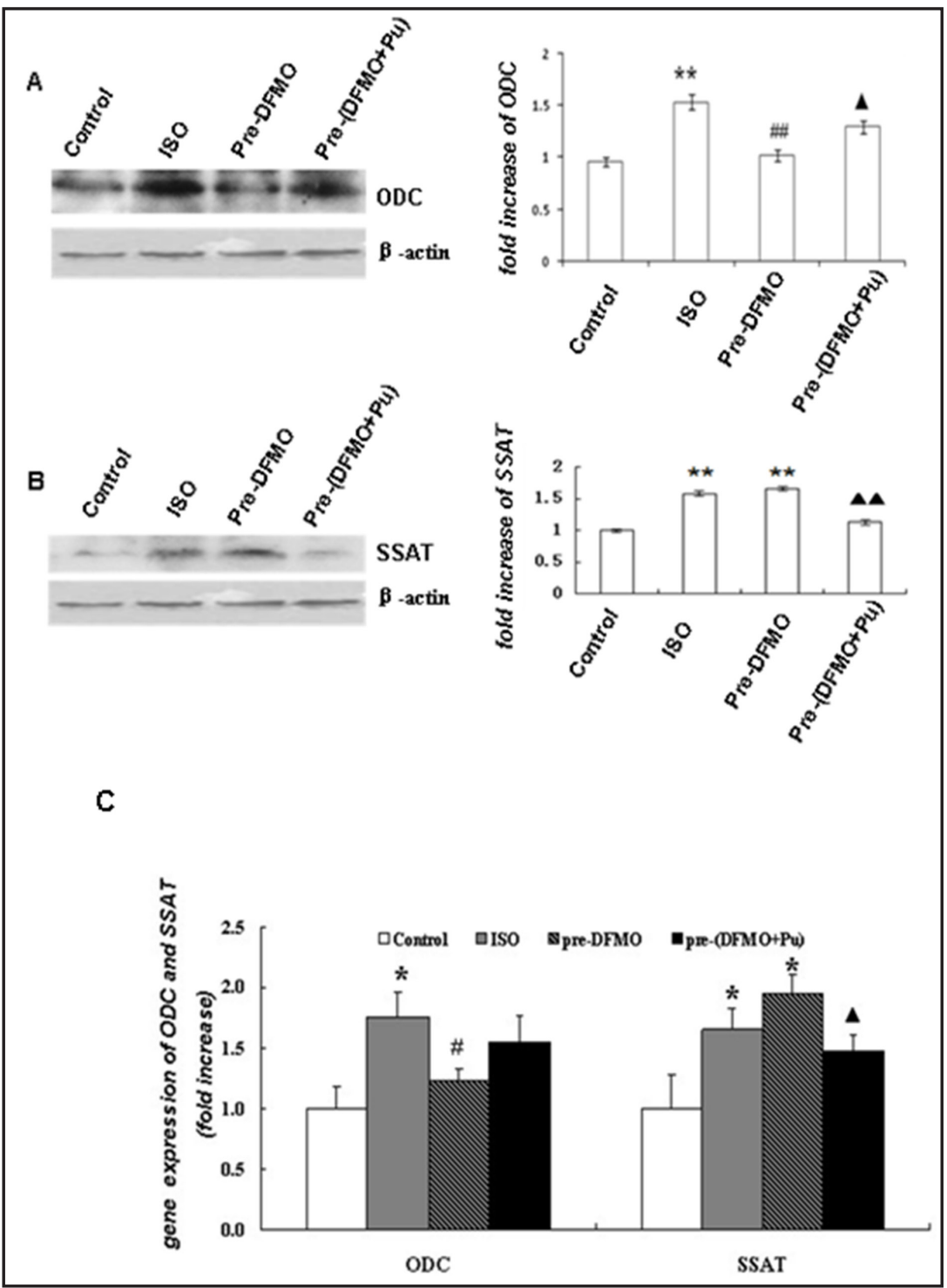

Table 1. LDH and MDA levels in culture medium. Data are means \pm S.E.M. of four separate experiments. ${ }^{\text {a }} P<0.01$ versus control group, ${ }^{\text {b }} P<0.05$ versus ISO group

\begin{tabular}{lcc}
\hline Group & LDH $(\mathrm{U} / \mathrm{mL})$ & MDA $(\mathrm{nmol} / \mathrm{mL})$ \\
\hline Control & $21.8 \pm 2.4$ & $7.6 \pm 0.9$ \\
ISO & $40.2 \pm 2.6^{\mathrm{a}}$ & $15.8 \pm 1.4^{\mathrm{a}}$ \\
Pre-DFMO $(0.5 \mathrm{mM})$ & $33.5 \pm 1.8^{\mathrm{b}}$ & $11.5 \pm 1.3^{\mathrm{b}}$ \\
Pre-(DFMO+Pu) $(0.5 \mathrm{mM})$ & $41.8 \pm 2.3$ & $16.1 \pm 1.1$ \\
\hline
\end{tabular}

to the ISO group, these morphological changes were less severe in the $0.5 \mathrm{mM}$ DFMO-treated group (Fig. 3A-D).

\section{DFMO inhibited ISO-induced LDH and MDA levels}

We measured the level of LDH and MDA of the culture medium to determine whether DFMO protects cardiomyocytes. Compared with the control group, the levels of LDH and MDA were significantly increased in the ISO group $(P<0.01)$. In the DFMO-pretreated group, LDH and MDA levels were significantly decreased $(P<0.05$ versus the ISO group). 


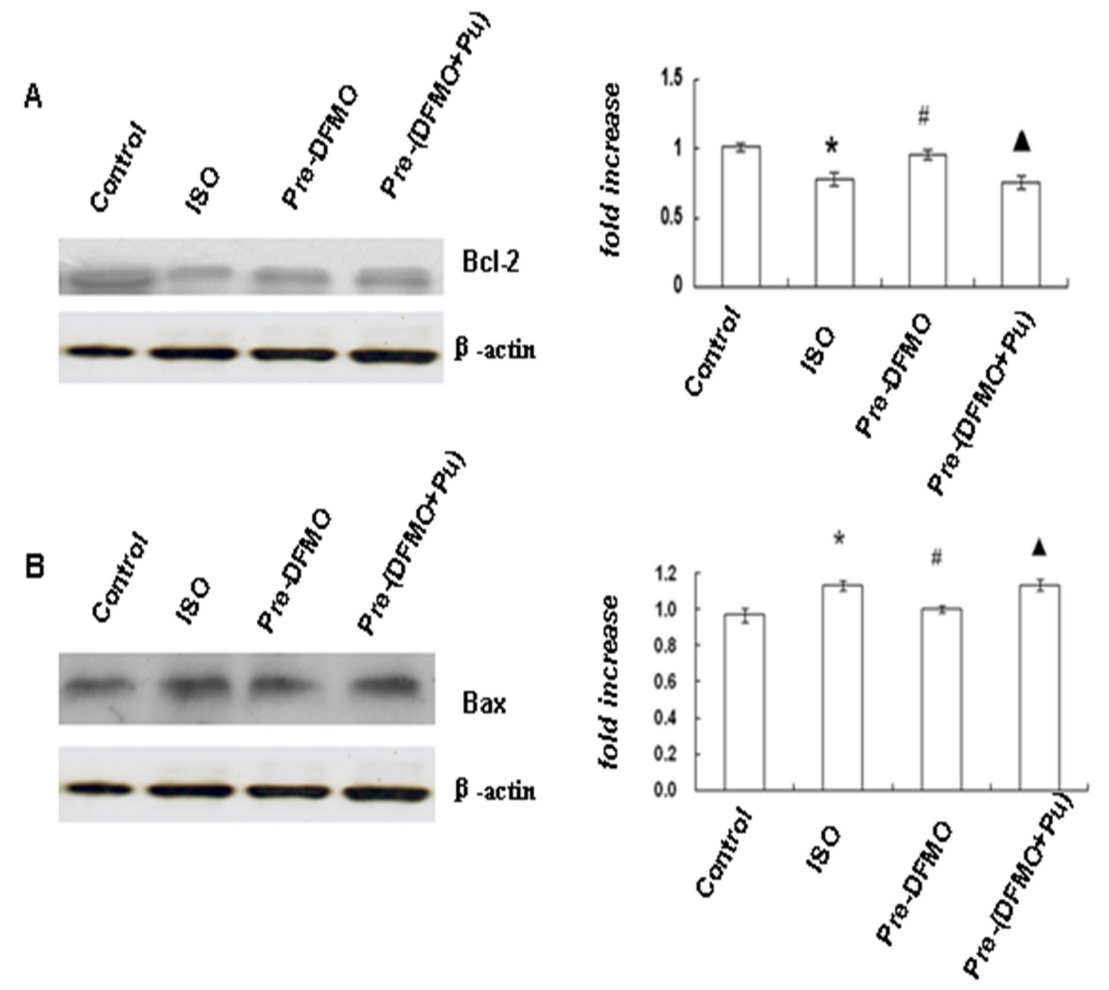

Fig. 5. DFMO reverses Bcl-2 and Bax expression patterns induced by ISO in neonatal rat cardiomyocytes. Bcl-2 (A) and Bax (B) protein levels were detected by Western blot. The control group's protein levels were considered basal levels, and data from the other experimental groups are expressed as fold change from the control group. The fold change values represent the mean \pm S.E.M. of four determinations. ${ }^{*} P<0.05$ versus control group. ${ }^{\#} P<0.05$ versus ISO group. ${ }^{\wedge} P<0.05$ versus $0.5 \mathrm{mM}$ pre-DFMO group.

No changes of LDH and MDA were observed in pre-(DFMO+Pu) group compared with the ISO group (Table 1).

The effect of DFMO on the gene and protein expression of ODC and SSAT

The gene expression of ODC and SSAT were increased significantly in the ISO group compared to the control group $(P<0.05)$, the gene expression of ODC was decreased by DFMO pretreatment. However, the gene expression of SSAT was increased in pre-DFMO group and pre-(DFMO + Pu) group. The protein expression of ODC and SSAT was increased in the ISO group $(P<0.01)$ and DFMO decreased the protein expression of ODC $(P<0.01)$. These changes were reversed by the addition of exogenous putrescine $(P<0.05$ or $P<0.01$ versus pre-DFMO group) (Fig. 4A-C).

The effect of DFMO on the protein expression of Bcl-2 and Bax

Bcl-2 is an anti-apoptotic factor, responsible for shifting the balance away from cell survival and toward cell apoptosis, and is involved in multiple forms of heart disease, The protein expression of Bcl-2 was decreased and the protein expression of Bax was increased in ISO group compared to the control group. Pretreatment with $0.5 \mathrm{mM}$ DFMO up regulated the expression of Bcl-2 and down regulated the expression of Bax when compared with the ISO group. Compared with the DFMO group, pretreatment with $0.5 \mathrm{mM}$ DFMO and $0.5 \mathrm{mM}$ Pu reversed the expression patterns of Bcl-2 and Bax (Fig. 5A and 5B, all $P<0.05$ ).

The effect of DFMO on the protein expression of GRP78, CHOP and cleaved caspase-12

To identify the direct effect of DFMO on ERS, the protein expression of GRP78, CHOP and cleaved caspase-12 were examined. Compared with the control group, ISO treatment 


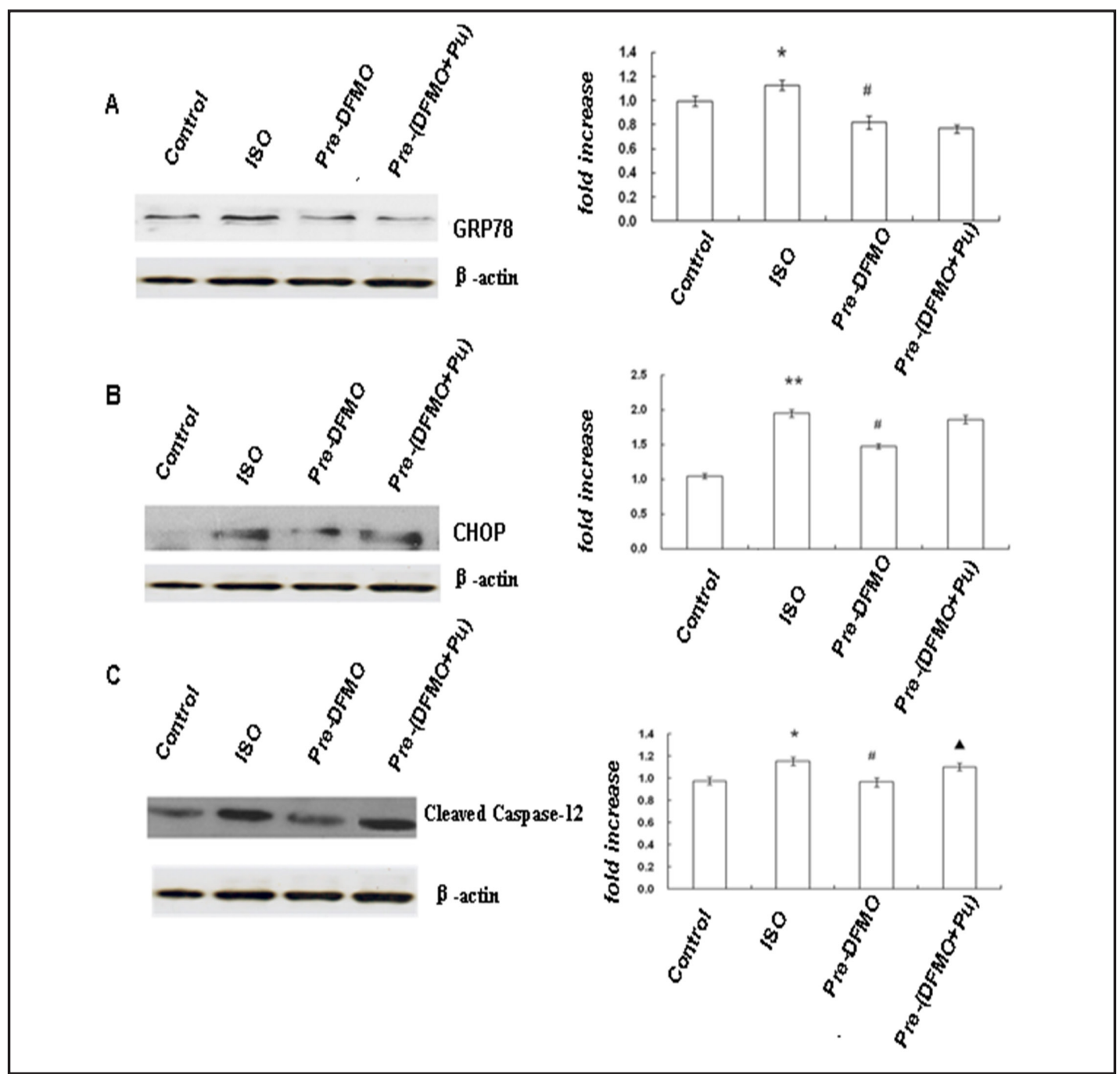

Fig. 6. DFMO inhibits ERS induced by ISO. Protein levels of ERS biomarkers GRP78 (A), CHOP (B), and cleaved caspase-12 (C) were determined by Western blot. The control group's protein levels were considered basal levels, and data from the other experimental groups are expressed as fold change from the control group. The fold change values represent the mean \pm S.E.M. of four determinations. ${ }^{*} P<0.05$ or $* * P<0.01$ versus the control group. ${ }^{\#} P<0.05$ versus ISO group. ${ }^{\wedge} P<0.05$ versus $0.5 \mathrm{mM}$ pre-DFMO group.

increased the expression levels of GRP78 $(P<0.05)$, CHOP $(P<0.01)$ and cleaved caspase-12 $(P<0.05)$. DFMO reversed these increases $(P<0.05$ versus ISO group). In contrast, the expression of GRP78 and CHOP were unchanged and the expression of cleaved caspase-12 was up regulated in the DFMO and Pu treatment group compared with the DFMO group $(P$ $<0.05$ ) (Fig. 6A-C).

\section{Discussion}

In response to pathophysiological stresses, cardiomyocytes undergo hypertrophic growth and/or apoptosis. Cardiac hypertrophy and apoptosis may play an important role in several cardiovascular diseases, such as ischemia/reperfusion, infarction, heart failure and aging. Naturally occurring polyamines are important cellular constituents essential for 
growth, differentiation, and apoptosis, with cellular levels tightly regulated. ODC, the first rate-limiting enzyme in polyamine biosynthesis, converts ornithine to putrescine, putrescine is then converted to spermidine and spermine. SSAT then acetylates both spermine and spermidine to generate acetylated polyamines, which are subsequently converted to putrescine by polyamine oxidase $[16,17]$.

Increasing evidence has showed that the level of ERS is significantly elevated in hypertrophic cardiomyocytes [18]. If the anti-hypertrophic role of polyamine depletion is mediated by ERS, is yet to be determined. In the present study, we demonstrated that DFMO can attenuate ISO-induced hypertrophy and apoptosis in cardiomyocytes. Moreover, these protective effects appear to be mediated by ERS, which may involve Bcl-2/Bax regulation.

Cardiac hypertrophy is characterized by an increase in cell surface area, protein synthesis and activation of the fetal gene program (e.g. ANP). Several recent studies have demonstrated that growth responses in cardiac tissue are accompanied by increased polyamine levels. In addition, DFMO, an irreversible inhibitor of ODC, can reduce putrescine levels by inhibiting the activity of ODC; it has also been shown to inhibit hypertrophy and fibrosis-related genic responses in cardiac cells [19-21]. In this study, we observed that ISO increased cardiac hypertrophy and the rate of apoptosis, and DFMO pretreatment attenuated hypertrophy and apoptosis in cardiomyocytes Furthermore, gene and protein levels of ODC and SSAT were increased in the ISO treated group. It is possible ISO may enhance polyamine synthesis through induction of ODC expression. It has been reported that $\beta$-adrenoceptor mediated hypertrophy in vivo occurs as a result of ODC induction [17]. It is also possible, however, that increased polyamines might influence cardiac hypertrophy and apoptosis through regulation of the ERS pathway. DFMO treatment did markedly decrease the gene and protein expression of ODC while increasing the gene and protein expression of SSAT. This effect was reversed by putrescine pretreatment. Previously our results have demonstrated that DFMO blocks polyamine synthesis through inhibiting the activity and expression of ODC, moreover, DFMO can decrease putrescine, spermidine and total polyamine pool. Our results have demonstrated that DFMO may play an anti-hypertrophic and anti-apoptotic role by inhibiting polyamine biosynthesis. These results are consistent with recent papers reporting a similar protective effect of DFMO against apoptosis in cardiac cells treated with aldosterone, or when cells were exposed to simulated ischemia conditions [20,21]. A study investigating the effects of polyamine depletion on norepinephrine mediated apoptosis in rat neonatal cardiomyocytes and $\mathrm{H}_{9} \mathrm{C}_{2}$ cardiomyoblasts demonstrated that DFMO had a prosurvival effect that was mediated through a specific network of phosphatases and kinases [22].

GRP78 is considered an ERS activation biomarker, and CHOP is the canonical protein in ERS-induced apoptosis. CHOP-deficient cells are resistant to ERS-mediated apoptosis and the overexpression of CHOP can lead to cell cycle arrest and/or apoptosis [23]. Enhanced expression of cleaved caspase-12 is an indicator of ERS associated apoptosis has been observed in heart tissues of diabetic patients [24]. The CHOP and caspase-12 proteins are distal effectors of ERS response mediating apoptotic signals. There is extensive evidence indicating ERS markers, GRP78 and CHOP are increased in cases of cardiac hypertrophy and incidences of heart failure $[25,26]$. ERS has been shown to lead to apoptosis in these processes and as a link between apoptosis, cardiomyocyte loss, ventricular remodeling, and deterioration of systolic performance in multiple experimental models [27, 28]. In this study we have demonstrated three critical points. One, we have shown that ISO induced cardiac hypertrophy in myocytes, this was accompanied by up regulation of GRP78, CHOP, and cleaved caspase-12. Two, DFMO down regulated the expression of GRP78, CHOP, and cleaved caspase-12. Three, the presence of exogenous putrescine reversed this DFMO-induced down regulation of ERS pathway markers. These findings suggest that DFMO inhibits the ERS pathway, thus attenuating cardiacmyocyte hypertrophy and apoptosis.

Many studies have demonstrated that overexpression of CHOP leads to decrease Bcl-2 protein levels and inhibits the translocation of Bax protein from the cytosol to the mitochondria [29]. Overexpression of Bcl-2 or Bax knockout has been shown to block CHOP- 
induced apoptosis [30]. Our experimental results have shown DFMO treatment to increase the expression of an anti-apoptotic gene (Bcl-2) and decrease the expression of a pro-apoptotic gene (Bax). These studies combined with our results suggests DFMO treatment inhibits ISOinduced cardiac hypertrophy and decreased expression of ERS markers, resulting in Bcl-2 and Bax expression changes, and subsequent decrease in apoptosis.

To our knowledge, this is the first study to assess the interaction between ODC mediated polyamine synthesis and ERS signaling in neonatal rat cardiomyocytes. These findings contribute to the elucidation of DFMO as a negative regulator in cardiac hypertrophy and the development of methodologies to explore therapeutics for cardiac diseases.

\section{Acknowledgements}

This research is supported by the National Natural Science Foundation of China (No. 81100170, 81100163, 81170178) and the Technology Research Foundation of the Department of Education of Heilongjiang Province (No. 12521617).

\section{Disclosure Statement}

The authors declare no conflict of interest.

\section{References}

-1 Zhao YJ, Xu CQ Zhang WH, Zhang L, Bian SL, Huang Q SunHL, Li QF, Zhang YQ Tian Y, Wang R, Yang BF, Li WM: Role of polyamines in myocardial ischemia/reperfusioninjury and their interactions with nitric oxide. Eur J Pharmacol 2007;562:236-246.

2 Tantini B, Fiumana E, Cetrullo S, Pignatti C, Bonavita F, Shantz LM, Giordano E, Muscari C, Flamigni F, Guamieri C, Stefanelli C, Caldarera CM: Involvement of polyamines in apoptosis of cardiac myoblasts in a model of simulated ischemia. J Mol Cell Cardiol 2006;40:775-782.

3 Minois N, Carmona-Gutierrez D, Madeo F: Polyamines in aging and disease. Aging 2011;3:716-732.

4 Anelli T, Sitia R: Protein quality control in the early secretory pathway. EMBO J 2008;27:315-327.

5 Kim I, Shu CW, Xu W, Shiau CW, Grant D, Vasile S, Cosford ND, Reed JC: Chemical biology investigation of cell death pathways activated by endoplasmic reticulum stress reveals cytoprotective modulators of ASK1. J Biol Chem 2009;284:1593-1603.

-6 Okada K, Minamino T, Tsukamoto Y, Liao Y, Tsukamoto O, Takashima S, Hirata A, Fujita M, Nagamachi Y, Nakatani T, Yutani C, Ozawa K, Ogawa S, Tomoike H, Hori M, Kitakaze M: Prolonged endoplasmic reticulum stress in hypertrophic and failing heart after aortic constriction: possible contribution of endoplasmic reticulum stress to cardiac myocyte apoptosis. Circulation 2004;110:705-712.

7 Thuerauf DJ, Marcinko M, Gude N, Rubio M, Sussman MA, Glembotski CC: Activation of the unfolded protein response in infarcted mouse heart and hypoxic cultured cardiac myocytes. Circ Res 2006;99:275-282. Qi X, Vallentin A, Churchill E, Mochly-Rosen D: $\delta$ PKC participates in the endoplasmic reticulum stressinduced response in cultured cardiac myocytes and ischemic heart. J Mol Cell Cardiol 2007;43:420-428. Glembotski CC: Endoplasimic reticulum stress in the heart. Circ Res 2007;101:975-984. Xu J, Zhou Q, Xu W, Cai L: Endoplasmic Reticulum Stress and Diabetic Cardiomyopathy. Exp Diabetes Res. 2012;2012:827971.

11 Drazner MH, Rame JE, Marino EK, Gottdiener JS, Kitzman DW, Gardin JM, Manolio TA, Dries DL, Siscovick DS: Increased left ventricular mass is a risk factor for the development of a depressed left ventricular ejection fraction within 5 years: the cardiovascular health study. J Am Coll Cardiol 2004;43:2207-2215.

12 Lin Y, Wang LN, Xi YH, Li HZ, Xiao FG, Zhao YJ, Tian Y, Yang BF, Xu CQ: L-arginine inhibits isoproterenolinduced cardiac hypertrophy through nitric oxide and polyamine pathways. Basic Clin Pharmacol Toxicol 2008;103:124-130. 


\section{Cellular Physiology $\quad$ Cell Physiol Biochem 2014;34:1455-1465

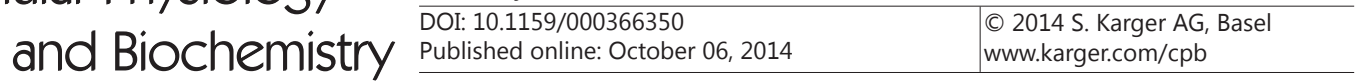 \\ Lin et al.: Polyamine Depletion Attenuates Hypertrophy and Endoplasmic Reticulum Stress in Cardiomyocytes}

13 Lin Y, Liu JC, Zhang XJ, Li GW, Wang LN, Xi YH, Li HZ, Zhao YJ, Xu CQ: Downregulation of the ornithine decarboxylase/polyamine system inhibits angiotensin-induced hypertrophy of cardiomyocytes through the NO/cGMP-dependent protein kinase type-I pathway. Cell Physiol Biochem 2010;25:443-450.

$\checkmark 14$ Lu FH, Fu SB , Leng XN, Zhang XY, Dong SY, Zhao YJ , Ren H , Li HL, Zhong X, Xu CQ Zhang WH: Role of the calcium-sensing receptor in cardiomyocyte apoptosis via the sarcoplamic reticulum and mitochondrial death pathway in cardiac hypertrophy and heart failure. Cell Physiol Biochem 2013;31:728-743.

-15 Zhao YJ, Zhang WH, Xu CQ Li HZ, Wang LN, Li H, Sun YH, Lin Y, Hang LP, Zhang L, Tian Y, Wang Rui, Yang BF, Li WM: Involvement of the ornithine decarboxylase/polyamine system in precondition-induced cardioprotection through an interaction with PKC in rat hearts. Mol Cel Biochem2009;332:135-144.

- 16 Fike JR, Gobbel GT, Chou D, Wijnhoven BP, Bellinzona M, Nakagawa M, Seilhan TM: Cellular proliferation and infiltration following interstitial irradiation of normal dog brain is altered by an inhibitor of polyamine synthesis. International journal of radiation oncology, biology, physics 1995;32:1035-1045.

17 Schluter KD, Frischfopf K, Flesch M, Rosenkranz S, Taimor G, Piper HM: Central role for ornithine decarboxylase in $\beta$-adrenoceptor mediated hypertrophy. Cardiovasc Res 2000;45:410-417.

18 Isodono K, Takahashi T, Imoto H, Nakanishi N, Ogata T, Asada S, Adachi A, Ueyama T, Oh H, Matsubara $\mathrm{H}$ : PARM-1 is an endoplasmic reticulum molecule involved in endoplasmic reticulum stress-induced apoptosis in rat cardiac myocytes. PLoS One 2010;5: e9746.

19 Tipnis UR, Chopra A, Campbell G, Boor P. Role of polyamines in atrial natriuretic peptide expression in isoproterenol-induced cardiac hypertrophy in rat. Biomedical Lett 1995;52:81-86.

20 Tipnis UR, He GY, Li S, Campbell G, Boor PJ: Attenuation of Isoproterenol-mediated Myocardial Injury in Rat by an Inhibitor of Polyamine Synthesis. Cardiovas Pathol 2000;9:273-280.

21 Cetrullo S, Facchini A, Stanic I, Tantini B, Pignatti C, Caldarera CM, Flamigni F: Difluoromethylornithine inhibits hypertrophic, profibrotic and pro-apoptotic actions of aldosterone in cardiac cells. Amino Acids 2010;38:525-531.

22 Cetrullo S, Tantini B, Facchini A, Pignatti C, Tefanelli C, Caldarera CM, Flamigni F: A pro-survival effect of polyamine depletion on norepinephrine mediated apoptosis in cardiac cells: role of signaling enzymes. Amino Acids 2011;40:1127-1137.

23 Fu HY, Okada K, Liao Y, Tsukamoto O, Isomura T, Asai M, Sawada T, Okuda K, Asano Y, Sanada S, Asanuma H, Asakura M, Takashima S, Komuro l, Kitakaze M, Minamino T: Ablation of C/EBP homologous protein attenuates endoplasmic reticulum-mediated apoptosis and cardiac dysfunction induced by pressure overload. Circulation 2010;122:361-369.

24 Dorn GW 2 $2^{\text {nd }}$ : Apoptotic and non-apoptotic programmed cardiomyocyte death in ventricular remodelling. Cardiovasc Res 2009;81:465-473.

-25 Zinszner H, Kuroda M, Wang X, Batchvarova N, Lightfoot RT, Remotti H, Stevens JL, Ron D: CHOP is implicated in programmed cell death in response to impaired function of the endoplasmic reticulum. Genes Dev 1998;12:982-995.

26 Zhang ZY, Liu XH, Hu WC, Rong F, Wu XD: The calcineurin-myocyte enhancer factor 2c pathway mediates cardiac hypertrophy induced by endoplasmic reticulum stress in neonatal rat cardiomyocytes. Am J Physiol Heart Circ Physiol 2010;298:H1499-1509.

27 Lee Y, Gustafsson AB: Role of apoptosis in cardiovascular disease. Apoptosis 2009;14:536-548.

28 Park CS, Cha H, Kwon EJ, Sreenivasaiah PK, Kim do H: The chemical chaperone 4-phenylbutyric acid attenuates pressure-overload cardiac hypertrophy by alleviating endoplasmic reticulum stress. Biochem Biophys Res Commun 2012;11;421:578-584.

29 McCullough KD, Martindale JL, Klotz LO, Aw TY, Holbrook NJ: Gadd153 sensitizes cells to endoplasmic reticulum stress by down-regulating Bcl-2 and perturbing the cellular redox state. Mol Cell Biol 2001;21:1249-1259.

30 Gotoh T, Terada K, Oyadomari S, Mori M: Hsp70-DnaJ chaperonepair prevents nitric oxide- and CHOPinduced apoptosis by inhibiting translocation of Bax to mitochondria. Cell Death Differ 2004;11:390-402. 